\title{
SURGERY ON KNOTS
}

\author{
W. B. RAYMOND LICKORISH
}

Abstract. Surgery on two distinct classical knots can create the same 3-manifold.

It is common knowledge, [5], [8], that every closed orientable 3-manifold can be obtained by performing surgery on a link in the 3 -sphere $S^{3}$. This means that any such 3-manifold bounds a 4-manifold which can be obtained by adding handles, of index two, to the 4-ball; the components of the link are the attaching spheres for the handles, a framing of the link defines the method of handle addition. (A more general process, which consists of removing many copies of $S^{1} \times D^{2}$ and replacing them via arbitrary homeomorphisms of $S^{1} \times S^{1}$, is called 'Dehn surgery'.) Rob Kirby has recently found a tangible equivalence relation, on the class of all framed links, with the property that two such links are equivalent if and only if they yield, after surgery, the same 3-manifold, [4]; (Robert Craggs [2] has a similar theory). Kirby asked if a single equivalence class could contain two different framed knots (links of but one component). The answer, given here, is 'yes'. The question, it should be noted, is a mild version of the 'Property P'-problem, [1], [7], which, in one form, asks whether the surgery function, that maps a framed knot to a 3-manifold, is injective in the sense that only the unknot maps to $S^{3}$.

THEOREM. There is a homology 3-sphere $M$ which can be obtained by surgery on either of two distinct knots.

Proof. Two presentations of a link $L$, with components $L_{1}$ and $L_{2}$, are shown in Figure 1. Each $L_{i}$ is unknotted and each is null-homotopic in the complement of the other. There is, however, asymmetry between the two components of $L$; in fact, $L$ was introduced to the author, by Joe Martin, as the simplest example for demonstrating the asymmetry of wrapping numbers.

Let each component of $L$ be allocated the framing -1 , and let $M$ be the corresponding 3-manifold produced by surgery. In the diagrams the orientation conventions are as follows: Let $N_{1}$ and $N_{2}$ be disjoint tubular neighbourhoods of $L_{1}$ and $L_{2}$, so that $M$ is obtained by removing the interiors of the $N_{i}$ and sewing back two copies of $S^{1} \times D^{2}$. For each $a \in S^{1}, a \times \partial D^{2}$ is identified with a curve in $\partial N_{i}$ which goes once around $\partial N_{i}$ longitudinally and once meridianally, screwing in a left-handed direction.

Now, because $L_{2}$ is unknotted, it is not necessary to use a link of two components to construct $M$. The process of removing $L_{2}$ and modifying $L_{1}$,

Received by the editors June 18, 1975.

AMS (MOS) subject classifications (1970). Primary 57A10; Secondary 55A25.

Copyright $\odot$ 1977, American Mathematical Society 

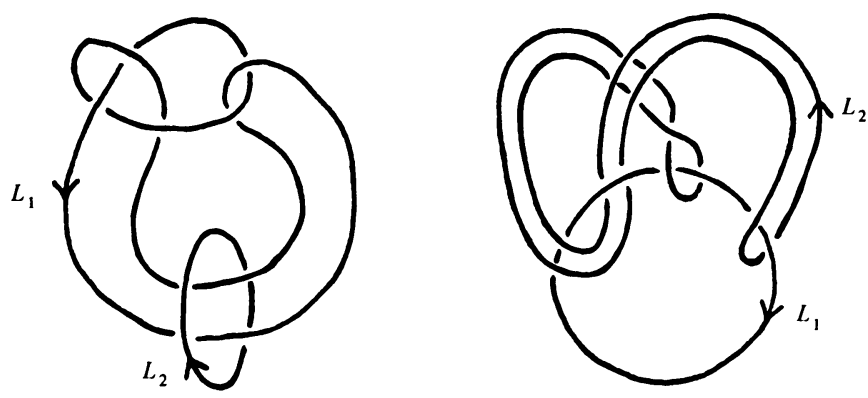

Figure 1

so as to create the same 3-manifold, was depicted by Hempel in [3]. Briefly, it is as follows. Cut $S^{3}-\stackrel{\circ}{N}_{2}$ along a disc whose boundary is in $N_{2}$, twist one side of the cut through a complete rotation, then glue together again. Assuming that the rotation was in the correct direction, it is now a meridianal curve in $\partial N_{2}$ which must needs be identified with $a \times \partial D^{2}$. This means that $N_{2}$ may be replaced whence it came, but the procedure has introduced a pair of cross-overs into $L_{1}$, which has now become a knot $K_{1}$ (see Figure 2). Hence, surgery on $K_{1}$, with framing -1 (because $L_{2}$ has linking number zero with $L_{1}$ ), yields $M$. By reversing the roles of $L_{1}$ and $L_{2}$, this argument also shows that $M$ can be obtained from the knot $K_{2}$.
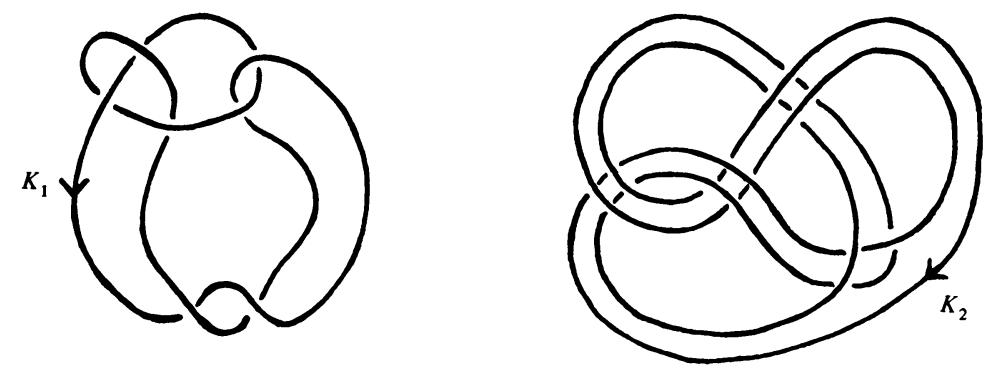

Figure 2

The plausible assertion that $K_{1}$ and $K_{2}$ be distinct can be verified by calculating that their Alexander polynomials are $\left(1-3 t+5 t^{2}-3 t^{3}+t^{4}\right)$ and $\left(1-t+t^{2}\right)$. It is, however, almost obvious from the surgical view of the Alexander polynomial, recently expounded by Dale Rolfsen [6], that the two polynomials must have different degrees.

The Kirby equivalence relation on links is best contemplated in terms of adding 2-handles to the 4-ball. The above example can, in that language, be regarded thus: Consider the 4-manifold obtained by adding a 2-handle to a 4-ball via $K_{1}$ with framing -1 . Add a 2-handle to the trivial knot with framing -1 (this being a permitted Kirby 'move'). Slide the first handle twice over the second so that the two attaching circles are now linked in link $L$. Slide the second handle four times over the first, and remove the first handle (which is now trivial) leaving the second handle added via $K_{2}$.

Dale Rolfsen reports that he can construct the lens space $L(23,7)$ by surgery on different knots, though one of his surgeries is a Dehn surgery. 


\section{REFERENCES}

1. R. H. Bing and J. M. Martin, Cubes with knotted holes, Trans. Amer. Math. Soc. 155 (1971), 217-231. MR 43 \#4018a.

2. R. Craggs, Stable representations for 3- and 4-manifolds (to appear).

3. J. P. Hempel, Construction of orientable 3-manifolds, Topology of 3-Manifolds and Related Topics (Proc. Univ. of Georgia Inst., 1961), Prentice-Hall, Englewood Cliffs, N.J., 1962, pp. 207-212. MR 25 \#3538.

4. R. C. Kirby, $A$ calculus for framed links in $S^{3}$ (to appear).

5. W. B. R. Lickorish, $A$ representation of orientable combinatorial 3-manifolds, Ann. of Math. (2) 76 (1962), 531-540. MR 27 \# 1929.

6. D. Rolfsen, A surgical view of Alexander's polynomial (Proc. Geometric Topology Conf., Utah, 1974), Lecture Notes in Math., vol. 438, Springer-Verlag, Berlin and New York, 1975, pp. 415-423. MR 50 \# 14751.

7. J. Simon, Some classes of knots with property P, Topology of Manifolds (Proc. Inst., Univ. of Georgia, Athens, Ga., 1969), Markham, Chicago, Ill., 1970, pp. 195-199. MR 43 \#4018b.

8. A. H. Wallace, Modifications and cobounding manifolds, Canad. J. Math. 12 (1960), 503-528. MR 23 \# A2887.

Department of Mathematics, Pembroke College, Cambridge, England 